\title{
Effects of dietary antioxidants on the immune function of middle-aged adults
}

\author{
David A. Hughes \\ Department of Nutrition, Diet and Health, Institute of Food Research, Norwich Research Park, Norwich NR4 7UA, UK
}

\begin{abstract}
The immune system is highly reliant on accurate cell-cell communication for optimal function, and any damage to the signalling systems involved will result in an impaired immune responsiveness. Oxidant-mediated tissue injury is a particular hazard to the immune system, since phagocytic cells produce reactive oxygen species as part of the body's defence against infection. Adequate amounts of neutralizing antioxidants are required, therefore, to prevent damage to the immune cells themselves. Many antioxidants can be obtained directly from the diet (e.g. ascorbic acid, $\alpha$-tocopherol, carotenoids and polyphenolic flavonoids) or require micronutrients as integral components (e.g. Se in the metalloenzyme glutathione peroxidase (EC 1.11.1.9)). Numerous epidemiological studies have found strong associations between diets rich in antioxidant nutrients and a reduced incidence of cancer, and it has been suggested that a boost to the body's immune system by antioxidants might, at least in part, account for this. Although more striking effects have been observed in the elderly, there is also evidence that antioxidant nutrients can modify cellmediated immune responses in younger individuals. Indeed, it might be essential to have an adequate intake of antioxidant nutrients from an early age in order to help prevent the development of, or at least delay the onset of, several degenerative disorders. The present paper will review the effects of specific nutrients on immune function in young to middle-aged human subjects, focusing on the antioxidant vitamins $\mathrm{C}$ and $\mathrm{E}$, and on Se. A further review, dealing more specifically with the effects of carotenoids on human immune function, will be presented at a forthcoming meeting of the Nutrition Society.
\end{abstract}

Antioxidants: Immune function: Vitamin C: Vitamin E

\section{Free radicals and reactive oxygen species}

Free radicals are highly-reactive molecules containing one or more unpaired electrons. Examples of free radicals are superoxide $\left(\mathrm{O}_{2}{ }^{-}\right)$and hydroxyl $(\mathrm{OH} \cdot)$. The term 'reactive oxygen species' (ROS) is a collective one that includes not only oxygen-centred radicals but also some non-radical derivatives of oxygen, such as $\mathrm{H}_{2} \mathrm{O}_{2}$, singlet oxygen and $\mathrm{HOCl} . \mathrm{H}_{2} \mathrm{O}_{2}$ can very easily break down, particularly in the presence of transition metal ions (e.g. $\mathrm{Fe}^{2+}$ ), to produce the hydroxyl radical, the most reactive and damaging of the oxygen free radicals:

$$
\mathrm{H}_{2} \mathrm{O}_{2}+\mathrm{Fe}^{2+} \rightarrow \mathrm{OH} \cdot+\mathrm{OH}^{-}+\mathrm{Fe}^{3+} .
$$

Free radicals are generated during normal cellular metabolism and are also made deliberately. For example, $\mathrm{O}_{2^{-}}-$ plays an essential role in the intracellular killing of micro- organisms by activated phagocytes. Free radical production in cells can be significantly increased by certain toxic 'redox-cycling' compounds such as various drugs and $\mathrm{CCl}_{4}$. Exogenous sources of free radicals include ozone, exposure to u.v. radiation in sunlight, and cigarette smoke.

All the major classes of biomolecules are vulnerable to free radical damage. Free radicals cause strand breaks in DNA (Halliwell \& Aruoma, 1991), which potentially can lead to subsequent misrepair and tumour cell formation. An example of free radical-mediated damage to proteins is the formation of cataracts, resulting from the damage to the crystallins in the lens of the eye. However, lipids are probably most susceptible to free radical attack, particularly long-chain polyunsaturated fatty acids which contain several double bonds. The oxidative destruction of polyunsaturated fatty acids, known as lipid peroxidation, can be extremely damaging, since it proceeds as a self-perpetuating chain reaction. 


\section{Antioxidant defences}

Since ROS are produced in vivo, organisms have evolved antioxidant defence systems either to prevent the generation of ROS or to intercept any that are produced. They exist in both the aqueous and membrane compartments of cells and can be enzymes or non-enzymes. Catalase ( $E C$ 1.11.1.6) and glutathione peroxidase (EC 1.11.1.9) are enzymes which can safely decompose peroxides, particularly $\mathrm{H}_{2} \mathrm{O}_{2}$ produced during the respiratory burst involved in microbial killing in phagocytic cells, whilst superoxide dismutase (EC 1.15.1.1) intercepts or 'scavenges' free radicals. Most free-radical scavengers are not enzymes, and many are obtained through the diet. In cell membranes the most important is $\alpha$-tocopherol, the major member of the vitamin $\mathrm{E}$ family. This molecule acts as a "chain-breaking antioxidant', intercepting lipid peroxyl radicals and so terminating lipid peroxidation chain reactions. Another group of lipid-soluble compounds that can act as antioxidants are the carotenoids, such as $\beta$-carotene, lycopene and lutein, found in highly-pigmented fruits and vegetables (Mangels et al. 1993). The polyene structure of these compounds allows the molecules to quench, or inactivate, singlet oxygen and free radicals. The major water-soluble free-radical scavenger is ascorbic acid (vitamin $\mathrm{C}$ ), which also plays a role in 'sparing' vitamin E, by regenerating $\alpha$-tocopherol from the oxidized tocopheroxyl radical (Bendich et al. 1986). More recently, attention has also focused on the antioxidant properties of plant polyphenols, found in tea and red wines (Rice-Evans, 1995), but considerably more information on the absorption, metabolism and excretion of these compounds in human subjects is required before their relative contribution to preventing oxidative damage can be assessed.

\section{Oxidative stress and the immune system}

Viewed from the perspective of a 'two-pan balance' with ROS in one pan and antioxidants in the other pan, it is thought that tipping the balance in favour of the ROS is a major contributor to several degenerative disorders such as cancer and cardiovascular diseases (Table 1) and to the ageing process in general. Striking improvements in immune function have been observed in elderly individuals following supplementation with antioxidant nutrients (see Lesourd, 1999), but there is growing evidence that effects can also be observed in younger healthy individuals. Strong associations between diets rich in antioxidant nutrients and a reduced incidence of cancer have been observed in numerous epidemiological studies, and it has been suggested that a boost to the body's immune system by

Table 1. Degenerative disorders associated with oxidative damage

\section{Cancer}

Cardiovascular disease

Stroke

Cataract

Degeneration of the macular area of the retina Immunosenescence

Ageing antioxidants might, at least in part, account for this finding. Indeed, it is probably crucial to attempt to keep the balance of ROS to antioxidants as level as possible, ideally by dietary means rather than by taking supplements, from as early an age as possible, in order to prolong, if not prevent, the onset of many age-related disorders.

The immune system is particularly sensitive to oxidative stress. Immune cells rely heavily on cell-cell communication, particularly via membrane-bound receptors, to work effectively. Cell membranes are rich in polyunsaturated fatty acids which, if peroxidized, can lead to a loss of membrane integrity, altered membrane fluidity (Baker \& Meydani, 1994), and result in alterations in intracellular signalling and cell function. It has been shown that exposure to ROS can lead to a reduction in cell-membrane receptor expression (Gruner et al. 1986). In addition, the production of ROS by phagocytic immune cells can damage the cells themselves if they are not sufficiently protected by antioxidants.

\section{Selenium}

$\mathrm{Se}$ is concentrated in tissues involved in the immune response, such as lymph nodes, spleen and liver (Spallholz et al. 1990), and various components of the immune system have been shown to be impaired if dietary intake of Se is inadequate (for review, see McKenzie et al. 1998). A major deficiency is seen in the microbicidal activity of phagocytes (Serfass \& Ganther, 1975) and this is thought to be due to the fact that $\mathrm{Se}$ is an integral component of glutathione peroxidase. Once a micro-organism has been engulfed into a phagocytic cell, the phagocytic vesicle fuses with lysosomes and the pathogen is destroyed by the respiratory burst, which generates ROS from molecular oxygen. It has been suggested that a lack of glutathione peroxidase can lead to damage of lysosomal membranes by lipid hydroperoxides, resulting in the release of various hydrolytic enzymes into the cytoplasm, with a subsequent reduction in cell function. Supplementation with Se appears to boost cell-mediated immune responses and, as well as protecting against oxidative damage, Se can also upregulate the expression of the high-affinity interleukin-2 receptor on $\mathrm{T}$ lymphocytes (Roy et al. 1994). This may, at least in part, explain the stimulatory effect of Se on B-cell antibody production, since this is regulated by T-cells.

In passing, it is worth mentioning the recent work of Beck and colleagues (Beck, 1998) who have demonstrated that Se-deficient mice infected with the coxsackievirus B3, develop an increased myocarditis compared with adequately-fed mice. The deficiency in Se was associated with a change to the viral genotype, converting the virus from a benign to a virulent strain (which involved six nucleotide changes). It is thought that this resulted from an enhanced ability of the virus to replicate in deficient hosts, thereby increasing the chances of a mutation occurring. This finding has obvious implications for populations containing individuals with poor nutritional status, since once the mutations have occurred even nutritionally-adequate hosts will be susceptible to viral-induced disease. 


\section{Ascorbic acid}

Vitamin C appears to affect most aspects of the immune system. It is found in high concentrations in leucocytes, it is rapidly utilized during infection, and reduced plasma levels are often associated with reduced immune function. Animal and human studies have suggested that the dietary requirements for vitamin $C$ are increased in cancer, surgical trauma and infectious diseases. However, the belief that high intakes of vitamin $\mathrm{C}$ will prevent the onset of the common cold has not been scientifically substantiated, although it is generally agreed that the associated symptoms following infection can be reduced by a moderate intake (Coulehan et al. 1974). Pauling's (1970) claims regarding the effects of vitamin $\mathrm{C}$ on the common cold certainly inspired a great deal of interest in the effect of this vitamin on immune function in the 1970s and early 1980s (for reviews, see Thomas \& Holt, 1978; Siegel, 1993), but research in this area has subsequently declined, and there have been very few recent studies examining the effects of vitamin $C$ on the immune system in healthy younger individuals. In one study, where a group of healthy young males was given $1-3 \mathrm{~g}$ vitamin $\mathrm{C} / \mathrm{d}$ for 3 weeks, there was an increase in neutrophil motility to lipopolysaccharide-activated autologous serum and in mitogen-stimulated lymphocyte proliferation following supplementation (Anderson et al. 1980). At the other end of the spectrum, to determine the effect of moderate vitamin C deficiency, Jacob et al. (1991) studied a group of young healthy non-smokers who were asked to consume an ascorbic acid-deficient diet which became gradually supplemented with vitamin $C$, starting at $5 \mathrm{mg} / \mathrm{d}$, and progressing up to $250 \mathrm{mg} / \mathrm{d}$. Ascorbic acid concentrations in plasma and leucocytes dropped initially to $<50 \%$ of baseline, and there was no change in lymphocyte proliferation. However, blood levels of glutathione and NAD(P) decreased during ascorbic acid depletion, as did the delayed hypersensitivity responsiveness to intradermal injection of seven recall antigens. On repletion, there was a recovery in delayed hypersensitivity response at intakes of 60 or $250 \mathrm{mg}$ vitamin C/d, but not in lymphocyte proliferation. Jacob et al. (1991) suggest that the inconsistency between these two tests regarding the influence of vitamin $C$ on cell-mediated immunity may result from higher sensitivity of the delayed hypersensitivity test, involvement of cells other than those isolated for in vitro cultures in the in vivo delayed hypersensitivity response, or other unknown factors. The lack of an effect on lymphocyte proliferation at an intake of $250 \mathrm{mg} / \mathrm{d}$ suggests that, at least in young individuals, only levels of vitamin $C$ that approach pharmacological doses can produce a quantifiable effect on this index of immune function. However, at present, there is no marker of immune function that is known to be indicative of a long-term beneficial effect in terms of reducing the incidence of degenerative disorders in later life. Although not an immunological study, one recent report provides an excellent example of the potential need to maintain adequate intakes of antioxidant nutrients in the middle years of life to prevent the accumulative damage caused by ROS being made manifest in later years. Jacques et al. (1997) examined the cross-sectional relationship between age-related lens opacities and vitamin C supplement use over a 10-12-year period in women without diagnosed cataract or diabetes. Use of vitamin $\mathrm{C}$ supplements for 10 years or more was associated with a $77 \%$ lower prevalence of early lens opacities and an $83 \%$ lower prevalence of moderate lens opacities compared with women who did not use supplements. Women who consumed vitamin $\mathrm{C}$ supplements for less than 10 years showed no evidence of a reduced prevalence of early opacities, suggesting that long-term consumption of vitamin $C$ supplements may substantially reduce the development of age-related lens opacities. While the use of supplements might be required to obtain sufficient intakes of vitamin $\mathrm{C}$ to prevent this form of oxidative damage, it is hoped that the intake required to maintain optimal immune function can be obtained from a healthy diet containing fruit and vegetables rich in antioxidants. This should be the case, since epidemiological studies of populations having a lower incidence of cancer suggest that the benefits are associated with the intake of increased amounts of these foodstuffs rather than the taking of supplements.

Vitamin $\mathrm{C}$ has been used to treat some clinical phagocytic cell dysfunctions. In Chediak-Higashi syndrome, which is characterized in part by defective neutrophil functions, vitamin $\mathrm{C}$ supplementation has been shown to increase neutrophil chemotaxis, improve bactericidal activity and reduce the length of clinical illness (Boxer et al. 1976). Vitamin $\mathrm{C}$ also appears to be beneficial in the treatment of chronic granulomatous disease (Anderson, 1982) and in recurrent pyogenic infections (Rebora et al. 1980).

Ascorbate provides important antioxidant protection to plasma lipids and lipid membranes (Frei et al. 1989) and can also neutralize phagocyte-derived oxidants released extracellularly (Anderson \& Lukey, 1987), thereby preventing oxidant-mediated tissue damage, particularly at sites of inflammatory activity. Other mechanisms which have been proposed for the immunostimulatory effects of vitamin $\mathrm{C}$ include: modulation of intracellular cyclic nucleotide levels, modulation of prostaglandin synthesis, enhancement of cytokine production, antagonism of the immunosuppressive interaction between histamines and leucocytes, the protection of 5-lipoxygenase (EC 1.13.1.34) (Anderson et al. 1990). There is a need for further research, not only into the mechanisms by which vitamin $\mathrm{C}$ can enhance immune cell function, but also to define the optimal levels of intake required to boost the immune system in younger individuals.

\section{Vitamin E}

Studies of human subjects and animals, in either states of deficiency or at supradietary levels, suggest strongly that $\alpha$-tocopherol is involved in maintaining immune cell function. Since vitamin $\mathrm{E}$ is the most effective chain-breaking lipid-soluble antioxidant present in cell membranes, it is considered likely that it plays a major role in maintaining cell membrane integrity by limiting lipid peroxidation by ROS. Vitamin E deficiency states are associated with depressed B-cell antibody production and T-cell proliferation to mitogenic stimulation, and an increased rate of infection. As with other dietary antioxidants, a marked improvement in immune indices can be seen in the elderly following supplementation with vitamin E (Meydani \& Beharka, 1996), but there is also evidence that increased 
intakes can modulate the function of immune cells in younger individuals. For example, supplementation with $300 \mathrm{mg}$ vitamin E/d depressed the bactericidal activity of leucocytes from a group of healthy young men (Prasad, 1980). This finding, together with the results of a study suggesting that prolonged high-dose intakes of $\alpha$-tocopherol $(1600 \mathrm{mg} / \mathrm{d})$ can lead to inhibition of neutrophil phagocytosis (Boxer, 1986), has led to the suggestion that perhaps vitamin $E$ supplements should only be taken in moderation to prevent an increased susceptibility to infection. However, paradoxically, a community-based survey reported a negative correlation between plasma vitamin $E$ levels and infectious disease episodes (Chavance et al. 1985), although this was in an elderly population.

Recently, Deveraj et al. (1996) examined the effect of high dose $\alpha$-tocopherol supplementation $(1200 \mathrm{mg} / \mathrm{d})$ on $e x$ vivo monocyte function. After 8 weeks of supplementation the in vitro release of ROS and lipid oxidation was decreased, both in the resting state and in lipopolysaccharide stimulated cells compared with both baseline and a 6-week washout period (when levels had returned to baseline). A similar effect was observed after culturing the cells in the presence of the protein kinase $\mathrm{C}$ inhibitor, Calphostin $\mathrm{C}$, suggesting that inhibition of the activity of this enzyme might be a mechanism by which $\alpha$-tocopherol can inhibit ROS release and lipid oxidation. Also, Deveraj \& Jialal (1997) reported a decrease in interleukin-1 $\beta$ release from resting and activated monocytes following vitamin $E$ supplementation, and have suggested that this inhibition is mediated in part by inhibition of 5-lipoxygenase, thereby decreasing leukotriene B4 levels, an agonist for interleukin$1 \beta$. It is also possible that vitamin $E$ and, indeed, other antioxidant nutrients can influence a variety of inflammatory processes by inhibiting the activity of the transcription factor nuclear factor $-\kappa \mathrm{B}$. This protein transcription factor is required for maximal transcription of many cytokines, including interleukin- $1 \beta$, and it is thought that the generation of ROS is a vital link in mediating nuclear factor- $k B$ activation by a variety of stimuli (Sen \& Packer, 1996; Jackson et al. 1998). Vitamin $\mathrm{E}$ might also down-regulate the positive feedback mechanism by which interleukin-1 $\beta$ can, in turn, activate nuclear factor- $\mathrm{KB}$ (Blackwell \& Christman, 1997).

Several studies have examined the effect of vitamin $E$ in cigarette smokers. Cigarette smoke contains millions of free radicals per puff, and other compounds present can stimulate the formation of other highly-reactive molecules (Pryor \& Stone, 1993). Serum levels of vitamin E (as well as vitamin $C$ and $\beta$-carotene) and lung vitamin $E$ concentrations are significantly lower in smokers compared with non-smokers (Bendich, 1994) and even supplementation with $2400 \mathrm{mg} / \mathrm{d}$ for 3 weeks failed to restore lung vitamin $\mathrm{E}$ levels to that found in non-smokers (Pacht et al. 1986). Circulating phagocytes from smokers produce high levels of free radicals, which probably in part accounts for the depressed immune function observed in smokers (Hughes et al. 1985; Johnson et al. 1990), and there is some evidence that vitamin $\mathrm{E}$ supplementation can reduce the overproduction of ROS by phagocytic cells from current smokers (Richards et al. 1990).
Reduced levels of vitamin $\mathrm{E}$ have been reported in human immunodeficiency virus-infected individuals. Passi et al. (1993) found that plasma vitamin E levels were significantly lower in a group of 200 human immunodeficiency viruspositive individuals compared with controls, but whether this is related to an inadequate intake of this vitamin is unclear. Dietary diaries from a group of 100 human immunodeficiency virus-infected asymptomatic men did not indicate an inadequate intake of vitamin $E$, but plasma levels were low or marginally low in $74 \%$ of the men (Beach et al. 1992). In a study of patients who had developed acquired immune deficiency syndrome an inverse relationship was observed between serum vitamin $E$ levels and severity of disease (Favier et al. 1994).

\section{Carotenoids}

The close association observed in epidemiological studies between high intakes of carotenoid-rich foodstuffs and a reduced incidence of cancer stimulated several studies of the effects of these compounds on immune function. Once again, emphasis has been given to studying effects in the elderly (for review, see Bendich, 1996), but there is also evidence that carotenoids can enhance immune function in healthy middle-aged individuals (Hughes et al. 1997). An associated review, dealing specifically with the effects of carotenoids on human immune function, will be presented at a forthcoming meeting of the Nutrition Society.

\section{Conclusions}

The present review has selected examples of studies demonstrating an effect of dietary antioxidants on the immune system of adult humans during mid-life. It is evident that there is a scarcity of studies performed in this age-group, particularly in healthy subjects. A major challenge in the area of nutritional immunology is to identify specific effects of dietary antioxidants on the immune system at this stage in life (when it is assumed to be functioning at an 'optimal' level) which are indicative of a beneficial effect that might only be seen in later years, in terms of a reduced incidence of age-related diseases.

\section{References}

Anderson R (1982) Effects of ascorbate on normal and abnormal leucocyte functions. International Journal for Vitamin and Nutrition Research 23, Suppl., 23-34.

Anderson R \& Lukey PT (1987) A biological role for ascorbate in the selective neutralisation of extracellular phagocyte-derived oxidants. Annals of the New York Academy of Sciences 498, 229 247.

Anderson R, Oosthuigen R, Maritz R, Theron A \& Van Rensburg A (1980) The effect of increasing weekly doses of ascorbate on certain cellular and humoral immune functions in normal volunteers. American Journal of Clinical Nutrition 33, 71-76.

Anderson R, Smith MJ, Joone GK \& Van Straden AM (1990) Vitamin $C$ and cellular immune functions. Protection against hypochlorous acid-mediated inactivation of glyceraldehyde-3phosphate dehydrogenase and ATP generation in human leukocytes as a possible mechanism of ascorbate-mediated 
immunostimulation. Annals of the New York Academy of Sciences 587, 34-48.

Baker KR \& Meydani M (1994) Beta-carotene in immunity and cancer. Journal of Optimal Nutrition 3, 39-50.

Beach RS, Mantero-Atienza E, Shor-Posner G, Javier JJ, Szapocznik J, Morgan R, Sauberlich HE, Cornwell PE, Eisdorfer C \& Baum MK (1992) Specific nutrient abnormalities in asymptomatic HIV-1 infection. AIDS 6, 701-708.

Beck MA (1998) The influence of antioxidant nutrients on viral infection. Nutrition Reviews 56, S140-S146.

Bendich A (1994) Recent advances in clinical research involving carotenoids. Pure and Applied Chemistry 66, 10171024.

Bendich A (1996) Antioxidant vitamins and human immune responses. Vitamins and Hormones 52, 35-62.

Bendich A, Burton GW, Machlin LJ, Scandurra O \& Wayner DDM (1986) The antioxidant role of vitamin C. Advances in Free Radical Biology and Medicine 2, 419-444.

Blackwell TS \& Christman JW (1997) The role of nuclear factor$\mathrm{KB}$ in cytokine gene regulation. American Joumal of Respiratory and Cell Molecular Biology 17, 3-9.

Boxer LA (1986) Regulation of phagocyte function by $\alpha$ tocopherol. Proceedings of the Nutrition Society 45, 333-334.

Boxer LA, Watanabe AM, Rister M \& Besch HR (1976) Correction of leucocyte function in Chediak-Higashi syndrome by ascorbate. New England Joumal of Medicine 295, 10411045.

Chavance M, Brubacher G, Herbeth B, Vernes G, Mistacki T, Deti F, Fournier C \& Janot C (1985) Immunological and nutritional status among the elderly. In Nutrition, Immunity, and Illness in the Elderly, pp. 137-145 [RK Chandra, editor]. New York: Pergamon Press.

Coulehan JL, Reisinger KS, Rogers KD \& Bradley DW (1974) Vitamin C prophylaxis in a boarding school. New England Journal of Medicine 290, 6-10.

Devaraj S \& Jialal I (1997) Alpha-tocopherol inhibits IL-1 $\beta$ from human monocytes. FASEB Joumal 11, 3753.

Devaraj S, Li D \& Jialal I (1996) The effects of $\alpha$-tocopherol supplementation on monocyte function. Journal of Clinical Investigation 98, 756-763.

Favier A, Sappey C, Leclerc P, Faure P \& Micoud M (1994) Antioxidant status and lipid peroxidation in patients infected with HIV. Chemico-Biological Interactions 91, 165-180.

Frei B, England L \& Ames BN (1989) Ascorbate is an outstanding antioxidant in human blood plasma. Proceedings of the National Academy of Sciences USA 86, 6377-6381.

Gruner S, Volk H-D, Falck P \& Baehr RV (1986) The influence of phagocytic stimuli on the expression of HLA-DR antigens; role of reactive oxygen intermediates. European Journal of Immunology 16, 212-215.

Halliwell B \& Aruoma OI (1991) DNA damage by oxygen-derived species: Its mechanisms and measurement in mammalian systems. FEBS Letters 281, 9-19.

Hughes DA, Haslam PL, Townsend PJ \& Turner-Warwick M (1985) Numerical and functional alterations in circulatory lymphocytes in cigarette smokers. Clinical and Experimental Immunology 61, 459-466.

Hughes DA, Wright AJA, Finglas PM, Peerless ACJ, Bailey AL, Astley SB, Pinder AC \& Southon S (1997) The effect of betacarotene supplementation on the immune function of blood monocytes from healthy male non-smokers. Journal of Laboratory and Clinical Medicine 129, 309-317.

Jackson MJ, McArdle A \& McArdle F (1998) Antioxidant micronutrients and gene expression. Proceedings of the Nutrition Society 57, 301-305.

Jacob RA, Kelley DS, Pianalto FS, Swendsein ME, Henning SM, Zhang JZ, Ames BN, Fraga CG \& Peters JH (1991)
Immunocompetence and oxidant defense during ascorbate depletion of healthy men. American Journal of Clinical Nutrition 54, 1302S-1309S.

Jacques PF, Taylor A, Hankinson SE, Willett WC, Mahnken B, Lee Y, Vaid K \& Lahav M (1997) Long-term vitamin C supplement use and prevalence of early age-related lens opacities. American Joumal of Clinical Nutrition 66, $911-$ 916.

Johnson JD, Houchens DP, Kluwe WM, Craig DK \& Fisher GL (1990) Effects of mainstream and environmental tobacco smoke on the inmune system in animals and humans: A review. $C R C$ Critical Reviews in Toxicology 134, 356-361.

Lesourd BM (1999) Immune response during disease and recovery in the elderly. Proceedings of the Nutrition Society 58, 85-98.

McKenzie RC, Rafferty TS \& Beckett GJ (1998) Selenium: an essential element for immune function. Immunology Today 19, 342-345.

Mangels AR, Holden JM, Beecher GR, Forman MR \& Lanza E (1993) Carotenoid content of fruits and vegetables: an evaluation of analytical data. Journal of the American Dietetic Association 93, 284-296.

Meydani SN \& Beharka AA (1996) Recent developments in vitamin $\mathrm{E}$ and immune response. Nutrition Reviews 66, S49S58.

Pacht ER, Kasek H, Mohammad JR, Cromwell DG \& Davis WB (1986) Deficiency of vitamin $E$ in the alveolar fluid of cigarette smokers: Influence on alveolar macrophage cytotoxicity. Journal of Clinical Investigation 77, 789-796.

Passi S, Picardo M \& Morrone A (1993) Study on plasma polyunsaturated phospholipids and vitamin $\mathrm{E}$, and on erythrocyte glutathione peroxidase in high risk HIV infection categories and AIDS patients. Clinical Chemistry and Enzymology Communications 5, 169-177.

Pauling L (1970) Vitamin C and the Common Cold. San Francisco, CA: W.H. Freeman.

Prasad JS (1980) Effect of vitamin E supplementation on leukocyte function. American Journal of Clinical Nutrition 33, 606-608.

Pryor WA \& Stone K (1993) Oxidants in cigarette smoke. Annals of the New York Academy of Sciences 686, 12-28.

Rebora A, Dallegri F \& Patrone F (1980) Neutrophil dysfunction and repeated infections: influence of levamisole and ascorbic acid. British Journal of Dermatology 21, 49-56.

Rice-Evans C (1995) Plant polyphenols: free radical scavengers or chain-breaking antioxidants? Biochemical Society Symposium 61, 103-116.

Richards GA, Theron AJ, Van Rensburg CEJ, Van Rensberg AJ, Van Der Merwe CA, Kuyl JM \& Anderson R (1990) Investigation of the effects of oral administration of vitamin $E$ and beta-carotene on the chemiluminescence responses and the frequency of sister chromatid exchanges in circulating leukocytes from cigarette smokers. American Review of Respiratory Disease 142, 648-654.

Roy M, Kiremidjian-Schumacher L, Wishe HI, Cohen MW \& Stotzky G (1994) Supplementation with selenium and human immune functions. I. Effect on lymphocyte proliferation and interleukin-2 receptor expression. Biological Trace Elements Research 41, 103-114.

Sen CK \& Packer L (1996) Antioxidant and redox regulation of gene transcription. FASEB Joumal 10, 709-720.

Serfass RE \& Ganther HE (1975) Defective microbicidal activity in glutathione peroxidase-deficient neutrophils of seleniumdeficient rats. Nature 255, 640-641.

Siegel BV (1993) Vitamin C and the immune response in health and disease. In Nutrition and Immunology, pp. 167-196 [DM Klurfeld, editor]. New York: Plenum Press. 
Spallholz JE, Boylan LM \& Larsen HS (1990) Advances in understanding selenium's role in the immune system. Annals of the New York Academy of Sciences 587, 123-139.
Thomas WR \& Holt PG (1978) Vitamin C and immunity: An assessment of the evidence. Clinical and Experimental Immunology 32, 370-379. 